\title{
L'emploi des femmes séropositives en France
}

\author{
Rosemary DRAY-SPIRA ${ }^{1}$
}

\begin{abstract}
Résumé
Dans ce chapitre, nous présentons plusieurs travaux issus d'analyses des données de la cohorte Anrs-C06-Primo et de l'enquête Anrs-Vespa, permettant de dresser un tableau de la situation de l'emploi des femmes séropositives en France et de décrire les mécanismes conduisant à cette situation. De la même façon que dans la population générale, parmi les personnes séropositives, les femmes ont un taux d'emploi significativement plus faible que les hommes. La situation professionnelle des femmes, plus précaire que celle des hommes, et qui s'ajoute à des charges domestiques et familiales souvent plus lourdes, apparaît plus déstabilisée par les effets de la maladie, en particulier quand celle-ci devient sévère. De plus, si certaines femmes, en particulier issues de l'immigration, parviennent à accéder à un emploi après le diagnostic du $\mathrm{VIH}$, elles n'ont cependant souvent accès qu'à des emplois précaires. L'infection par le VIH elle-même, mais aussi les caractéristiques sociodémographiques et professionnelles pèsent de façon concomitante sur la situation des personnes atteintes et rendent les femmes séropositives particulièrement exposées aux difficultés d'emploi.
\end{abstract}

Mots-clés : taux d'emploi, perte d'emploi, accès à l'emploi, enquête Vespa, cohorte Primo.

L'emploi est un facteur majeur de maintien du niveau de ressources et des conditions de vie, en particulier parmi les personnes atteintes d'affections de longue durée [1]. En outre, il a été montré que l'absence d'emploi constituait un facteur de risque indépendant de la morbidité et de la mortalité par suicide et mort violente [2, 3-5].

Bien que l'infection par le VIH affecte principalement des adultes en âge de travailler, différents travaux ont mis en évidence dans les pays occidentaux des taux de non-emplois élevés (entre 45 et $65 \%$ ) parmi les personnes vivant avec le $\mathrm{VIH} /$ sida à l'ère des thérapies antirétrovirales hautement actives ${ }^{2}$ [6-16]. Cependant, ces travaux ne permettent pas de déterminer dans quelle mesure de tels taux résultent d'un éventuel impact de l'infection par le VIH sur l'emploi, de caractéristiques défavorables sur le marché de l'emploi préexistantes à la survenue de la maladie, ou bien d'une combinaison de ces deux phénomènes.

Le fait d'être une femme constitue en lui-même un désavantage au regard de l'emploi au sein de la population générale française

\footnotetext{
Unité Inserm 687, hôpital national de Saint-Maurice.

2 Traduction française de Highly active antiretroviral therapy (HAART) : multithérapie destinée à obtenir une diminution maximale de la charge virale.
}

[17]. De plus, parmi les personnes séropositives, les femmes sont plus souvent originaires d'un autre pays que la France, ont un niveau d'éducation plus faible et sont plus jeunes comparées aux hommes, autant de caractéristiques qui constituent des désavantages supplémentaires au regard de l'emploi. Les femmes vivent également plus souvent que les hommes en familles monoparentales, avec les difficultés que cela engendre pour supporter à la fois les charges familiales et professionnelles. De telles difficultés sont probablement encore accentuées dans le cas d'une affection chronique telle que la maladie à VIH, suggérant que, dans ce champ, la situation des femmes séropositives pourrait être particulièrement défavorable.

Nous présentons ici plusieurs travaux issus d'analyses des données de la cohorte Anrs-C06-Primo et de l'enquête Anrs-Vespa ${ }^{3}$, permettant de dresser un tableau de la situation de l'emploi des femmes séropositives en France et de décrire les mécanismes conduisant à cette situation par comparaison avec d'une part les femmes de la population générale et, d'autre part, les hommes séropositifs.

\section{Les enquêtes et leur méthodologie}

\section{La cohorte Anrs-C06-Primo}

Primo est une cohorte prospective observationnelle ouverte, incluant depuis novembre 1996 des patients au cours ou au décours immédiat du stade de primo-infection au VIH, c'est-àdire au cours des semaines suivant la contamination par le virus. II s'agit d'une étude multicentrique réalisée en France métropolitaine et dans les départements d'Outre-Mer [18]. Après l'inclusion, les patients sont revus en consultation à l'hôpital à un mois, à trois mois, à six mois, puis tous les six mois par la suite. À la date du 31 décembre 2002, 319 patients avaient été inclus et suivis au moins six mois au sein de la cohorte (2,5 ans de durée médiane de suivi).

Cette étude avec bénéfice individuel direct ${ }^{4}$ a été organisée d'emblée de manière à être multidisciplinaire, en réunissant des cliniciens, des virologues, des immunologistes et des épidémiologistes. L'objectif principal de la cohorte Primo est d'ordre clinicobiologique. De plus, l'étude vise à décrire l'évolution des

\footnotetext{
Également appelée Anrs-EN12-Vespa.

4 Étude coordonnée par l'unité Inserm 822 (L Meyer) et financée par l'Anrs,
} 
principales caractéristiques de la situation sociale des individus au cours de la maladie à VIH et à étudier les relations entre ces caractéristiques sociales et l'évolution de l'état de santé au cours du temps.

\section{L'enquête Anrs-Vespa}

L'enquête Vespa est une large enquête transversale nationale effectuée entre décembre 2002 et septembre 2003 en milieu hospitalier au sein des services spécialisés dans la prise en charge du VIH en France métropolitaine (voir encadré sur la description détaillée de l'enquête, page 215). Son objectif principal était de décrire la situation sociale et les conditions de vie des personnes vivant avec le $\mathrm{VIH} /$ sida en France à l'ère des thérapies antirétrovirales [19]. Afin d'être en mesure d'extrapoler les résultats de l'enquête à l'ensemble de la population des personnes vivant avec le VIH et suivies à l'hôpital en France, une procédure de pondération et de redressement utilisant une méthode de calage sur marges a été effectuée. Les résultats présentés ici portent tous sur ces données redressées de l'enquête.

\section{Taux d'emploi des femmes séropositives en France en 2002-2003 : comparaison avec les femmes de la population générale et avec les hommes séropositifs}

Le taux d'emploi des personnes séropositives en France en 2002 2003 a été estimé à partir des données de l'enquête Vespa. La méthode de la standardisation directe a été utilisée pour estimer les taux d'emploi standardisés parmi les personnes infectées par le VIH, en considérant l'échantillon de l'enquête emploi 2002 de l'Insee ( $n=34423$ sujets âgés de 16 à 59 ans) représentatif de la population générale française comme population de référence. En d'autres termes, nous avons calculé les taux d'emploi qui seraient attendus parmi les femmes et les hommes infectés par le VIH si ces personnes étaient réparties de la même façon que, respectivement, les hommes et les femmes de la population générale en termes d'âge, de nationalité et de niveau d'éducation. Dans la mesure où les conséquences de la maladie sur l'emploi ont probablement été profondément modifiées par les changements survenus dans la prise en charge de la maladie, l'écart de taux d'emploi entre les personnes séropositives et la population générale a été calculé séparément selon la période de diagnostic de la maladie. Environ un tiers des patients de l'enquête Vespa avait été diagnostiqué à partir de 1996 (année d'introduction des traitements antirétroviraux hautement actifs), ce qui représentait un effectif insuffisant pour conduire l'analyse au sein de ce sous-groupe seul. Nous avons donc considéré que les patients infectés par le VIH diagnostiqués à partir de 1994 avaient davantage bénéficié de l'effet de ces nouveaux traitements que ceux diagnostiqués auparavant, et les analyses ont été conduites séparément selon que la période du diagnostic d'infection à VIH était ou non antérieure à 1994.

Alors que le taux d'emploi des femmes de la population générale française atteint $58,4 \%$, les taux d'emploi standardisés des femmes séropositives n'atteignent respectivement que $42,6 \%$ (intervalle de confiance à $95 \%$ : [32,6\%; $48,5 \%]$ ) pour celles diagnostiquées avant 1994 , et $52,3 \%$ [47,4 \%; $58,7 \%]$ pour celles diagnostiquées à partir de 1994. Au sein de chacune de ces trois sous-populations, le taux d'emploi des femmes est systématiquement plus faible que celui des hommes (figure 1).

L'écart de taux d'emploi entre les femmes séropositives et les femmes de la population générale atteint respectivement $27 \%$ [17\%; $44 \%$ ] pour celles diagnostiquées avant 1994 et $10 \%$ $[-1 \% ; 19 \%]$ pour celles diagnostiquées à partir de 1994. L'ampleur de ces écarts de taux d'emploi avec la population générale française est comparable pour les hommes séropositifs avec $23 \%[15 \% ; 30 \%]$ et $8 \%[4 \% ; 13 \%]$ respectivement pour ceux diagnostiqués avant et à partir de 1994 (figure 2).

Ces données suggèrent qu'en France, le taux d'emploi des femmes séropositives est plus faible que celui des femmes de la population générale, même en tenant compte des différences dans les caractéristiques sociodémographiques de ces deux populations. De façon logique, l'écart de taux d'emploi avec la population générale apparaît plus marqué pour les femmes diagnostiquées avant 1994, c'est-à-dire celles dont la maladie a eu le temps de progresser avant qu'elles ne puissent bénéficier des traitements antirétroviraux hautement actifs, que parmi celles qui ont été diagnostiquées plus tardivement. Néanmoins, même parmi les femmes infectées par le VIH qui ont eu accès à ces traitements précocement dans l'histoire de leur maladie, le taux d'emploi en 2003 avait tendance à être plus faible que dans la population générale. Cette différence persistante pourrait être expliquée par un effet propre de la maladie à VIH sur l'emploi, qui pourrait résulter de deux phénomènes complémentaires : d'une part, parmi les personnes qui travaillent, un risque de perte d'emploi plus important pour les personnes séropositives que dans la population générale; d'autre part, parmi les personnes qui ne travaillent pas, des difficultés d'accès ou de retour à l'emploi accentuées en cas d'infection par le VIH. Cependant, les données disponibles ne permettent pas de comparer ces phénomènes de perte et d'accès à l'emploi entre les femmes séropositives et celles de la population générale en France.

Par ailleurs, il apparaît que l'écart de taux d'emploi entre les personnes séropositives et la population générale est du même ordre pour les hommes et pour les femmes, suggérant un impact de la maladie sur l'emploi d'ampleur comparable entre les deux sexes. Cependant, le taux d'emploi mesuré à un moment donné résulte de processus dynamiques d'entrées et sorties du travail et on ne peut pas exclure, à partir de ces données, qu'il existe des différences hommes/femmes dans de tels processus dynamiques. C'est pourquoi, les travaux présentés par la suite ont 
Figure 1

Taux d'emploi standardisés* de la population générale française et des 2750 participants de l'enquête Vespa âgés de moins de 60 ans, selon le sexe et la période du diagnostic du VIH

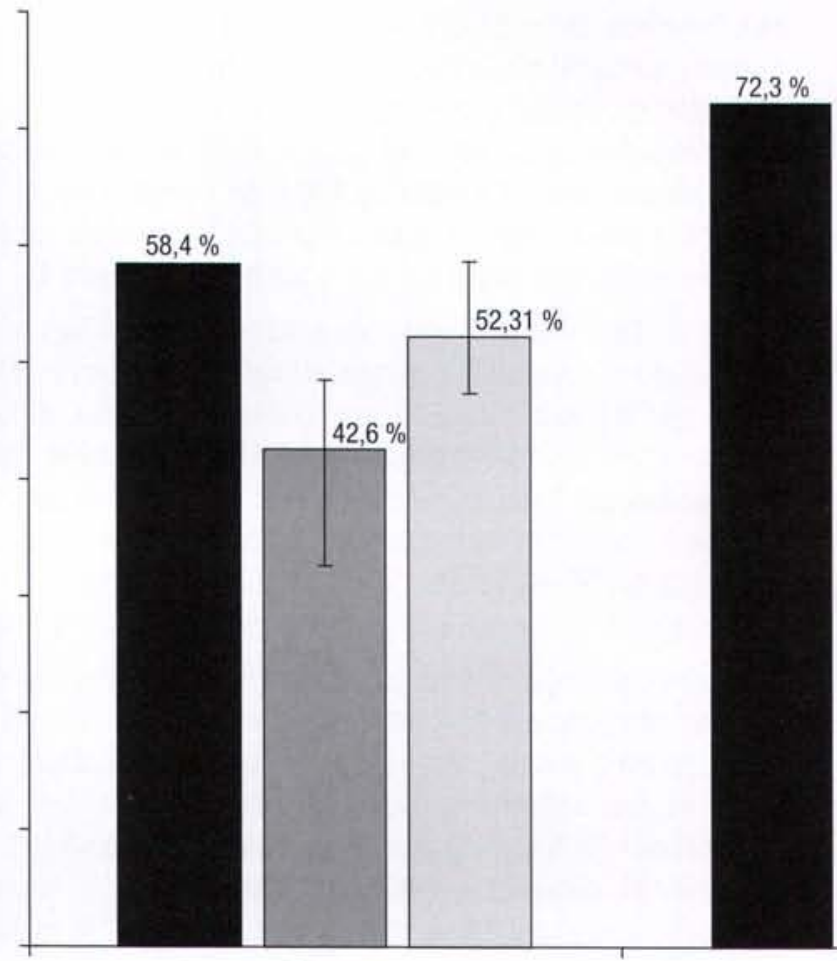

Femmes

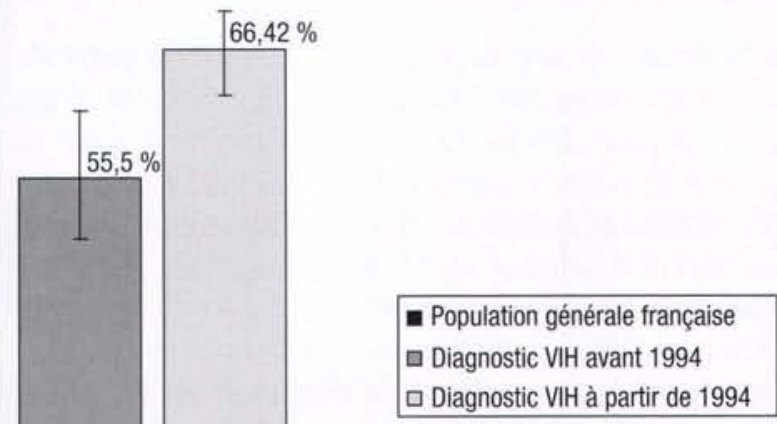

Hommes

* Taux d'emploi standardisé sur l'âge, le sexe, la nationalité et le niveau d'éducation.

porté, de façon plus spécifique, sur les processus de perte d'emploi et d'accès/retour à l'emploi parmi les femmes et les hommes infectés par le VIH.

\section{Risque de perte d'emploi au cours de la maladie à VIH}

Les données de la cohorte Primo permettent d'estimer le risque de perte d'emploi au cours de cette maladie et d'en étudier les déterminants. En effet, elles comportent, pour chacun des participants, des informations sur la situation d'emploi au moment de l'inclusion dans la cohorte, qui correspond à la période précoce de la primo-infection par le VIH, puis tout au long de son suivi dans la cohorte. La perte d'emploi a été définie comme le passage d'une situation d'emploi à une situation de non-emploi entre deux visites semestrielles de suivi. L'identification des caractéristiques associées au risque de perte d'emploi a été effectuée en comparant les périodes de six mois séparant deux visites de cohorte au cours desquelles il y avait eu une perte d'emploi à celles au cours desquelles l'emploi avait été maintenu. Des modèles marginaux de régression logistique uni et multivariés (méthode des équations estimées généralisées, GEE) ont été utilisés. Les covariables incluses dans les analyses étaient constituées : 1) de caractéristiques fixes au cours du temps recueillies au moment de l'inclusion (sexe, pays de naissance, niveau d'éducation, catégorie socioprofessionnelle, mode de logement, taux de CD4 et charge virale plasmatique à l'inclusion, co-morbidité chronique, usage de drogues) ; 2) de caractéristiques actualisées au moment de la visite de suivi, marquant le début de chaque observation, variables dépendantes du temps (âge, statut d'emploi, existence d'une relation affective stable, taux de $\mathrm{CD} 4$, charge virale plasmatique, stade clinique, survenue d'au moins une hospitalisation depuis la visite précédente, et co-morbidité aiguë).

Parmi les patients de la cohorte Primo qui avaient un emploi, globalement presque un sur cinq $(18,0 \%)$ avait perdu son emploi au moins une fois au cours d'une durée médiane de suivi de 2,5 ans après la survenue de l'infection à VIH. Cette proportion atteignaient $38,6 \%$ chez les femmes.

Ce risque de perte d'emploi au cours du suivi apparaît significativement plus élevé pour les femmes que pour les hommes, et ce, indépendamment des caractéristiques majeures de la position sur le marché de l'emploi et de l'état de santé (tableau 1). 
Figure 2

Ecart de taux d'emploi` par rapport à la population générale parmi les 2750 participants de l'enquête Vespa âgés de moins de 60 ans, selon le sexe et la période du diagnostic du VIH

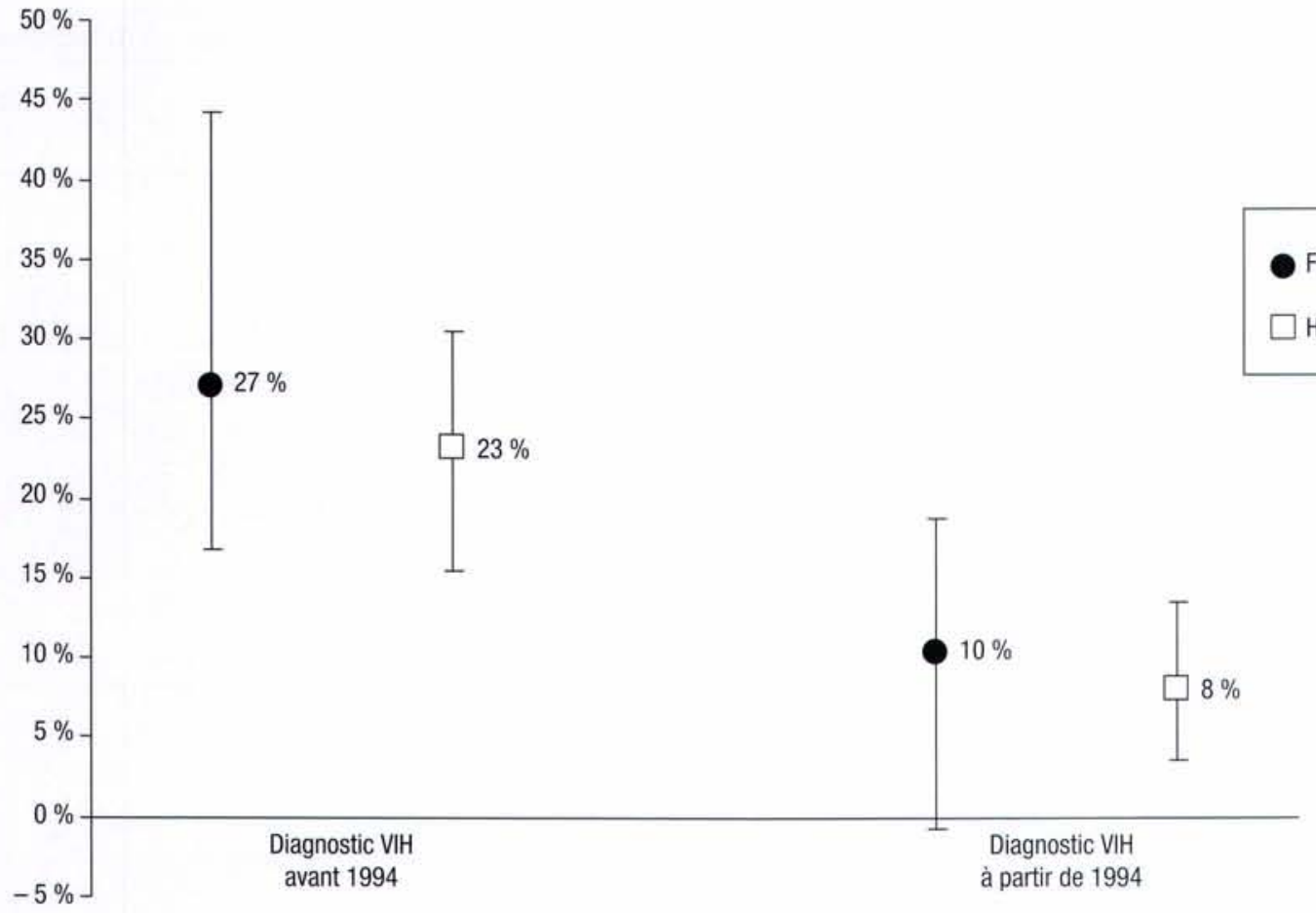

* Taux d'emploi standardisé sur l'âge, le sexe, la nationalité et le niveau d'éducation.

En effet, en analyse multivariée, le risque de perte d'emploi est associé aux caractéristiques de la situation socio-économique des patients : le fait d'être une femme (odds ratio ajusté : 3,1 ; intervalle de confiance à $95 \%:[1,1 ; 8,5])$, d'occuper un emploi précaire $(3,8[1,5 ; 9,3])$ et d'être hébergée chez des amis au moment de l'inclusion $(4,2[1,6 ; 11,2])$ constitue un facteur de risque indépendant de perte d'emploi ; les sujets appartenant à une catégorie socioprofessionnelle élevée ont un risque de perte d'emploi significativement plus faible que les autres. En outre, indépendamment des caractéristiques socio-économiques, le risque de perte d'emploi est plus élevé parmi les individus en mauvais état de santé : une infection à VIH avancée, comme en témoignent une charge virale actualisée dans le temps de plus de 10000 copies/ml, soit de façon persistante $(2,4[1,1 ; 5,0])$, soit incidente $(3,7[1,0 ; 13,9])$, et la survenue d'une hospitalisation au cours des six mois précédents $(3,9[1,6 ; 9,7])$ constituent des facteurs de risque indépendants de perte d'emploi ; un taux de CD4 inférieur à $350 / \mathrm{mm}^{3}$ à l'inclusion $(1,9[0,9 ; 4,3])$ et une comorbidité chronique à l'inclusion $(1,8[0,9 ; 3,6])$ tendent également à être associés à un risque augmenté de perte d'emploi.

Les données de l'enquête Vespa renseignent de façon rétrospective sur la trajectoire d'emploi entre le moment du diagnostic et 2003. Parmi les 478 participants diagnostiqués à l'ère des traitements antirétroviraux et qui avaient un emploi au moment du diagnostic du VIH, globalement, $26,4 \%$ ne travaillaient plus en 2003. Cette proportion atteint $39,3 \%$ parmi les femmes $(34,2 \%$ parmi les Françaises et $51,2 \%$ parmi les migrantes), versus $23,1 \%$ parmi les hommes (22,8 \% parmi les Français et 25,0 \% parmi les migrants). Ainsi, les données de l'enquête Vespa suggèrent, de la même façon que les données de Primo, que les femmes, qu'elles soient originaires de France ou d'un autre pays, ont un risque de perte d'emploi au cours de la maladie à VIH plus élevé que les hommes (hazard ratio ajusté : 1,6; intervalle de confiance à $95 \%:[1,1 ; 2,4])$.

De plus, les données de l'enquête Vespa permettent d'analyser plus avant ces différences. Ainsi, les patients ayant perdu leur emploi entre le diagnostic et 2003 ont été comparés quant à la sévérité de la maladie (mesurée par la progression vers le stade sida ou par un taux de CD4 $<200 / \mathrm{mm}^{3}$ ) et à l'expérience de discriminations dans l'emploi à ceux l'ayant conservé, en utilisant des modèles de Cox ajustés sur les caractéristiques sociodémographiques et professionnelles. Ces analyses suggèrent que le " sur-risque " féminin de ne pas rester en emploi au cours de la maladie pourrait être lié à un impact plus marqué de la progression de l'infection à VIH sur la situation d'emploi pour les femmes que pour les hommes. En effet, une maladie sévère, 
Tableau 1

Caractéristiques associées au risque de non-maintien dans l'emploi en analyse multivariée* parmi les $\mathbf{2 8 3}$ participants de la cohorte Primo, qui avaient un emploi lors d'au moins une visite de cohorte

\begin{tabular}{|c|c|c|}
\hline & Odds ratio ajusté & Intervalle de confiance à $95 \%$ \\
\hline $\begin{array}{l}\text { Sexe } \\
\text { Hommes } \\
\text { Femmes }\end{array}$ & $\begin{array}{l}1 \\
3,1^{\star \star}\end{array}$ & $1,1-8,5$ \\
\hline $\begin{array}{l}\text { Catégorie socioprofessionnelle à l'inclusion } \\
\text { Elevée (artisans, commerçants, chefs d'entreprise, cadres) } \\
\text { Intermédiaire (professions intermédiaires) } \\
\text { Basse (employés, ouvriers, agriculteurs) } \\
\text { Inactivité / Indéterminée }\end{array}$ & $\begin{array}{l}1 \\
5,1^{\star \star} \\
4,3^{\star \star} \\
5,1^{\star *}\end{array}$ & $\begin{array}{l}1,0-25,9 \\
1,1-17,1 \\
1,3-21,0\end{array}$ \\
\hline $\begin{array}{l}\text { Statut d'emploi } \\
\text { Emploi stable } \\
\text { Emploi précaire }\end{array}$ & $\begin{array}{l}1 \\
3,8^{* *}\end{array}$ & $1,5-9,3$ \\
\hline $\begin{array}{l}\text { Hébergement chez des amis à l'inclusion } \\
\text { Non } \\
\text { Oui }\end{array}$ & $\begin{array}{l}1 \\
4,2^{\star \star}\end{array}$ & $1,6-11,2$ \\
\hline $\begin{array}{l}\text { Taux de CD4 /mm3 à l'inclusion } \\
\geqslant 350 \\
<350\end{array}$ & $\begin{array}{l}1 \\
1,9^{\star \star \star}\end{array}$ & $0,9-4,3$ \\
\hline $\begin{array}{l}\text { Charge virale plasmatique actualisée (copies/ml) } \\
\leqslant 10000 \\
>10000 \text { persistante } \\
>10000 \text { incidente } \\
\text { Indéterminée }\end{array}$ & $\begin{array}{l}1 \\
2,4^{\star \star} \\
3,7^{\star \star} \\
0,7\end{array}$ & $\begin{array}{l}1,1-5,0 \\
1,0-13,9 \\
0,1-10,3\end{array}$ \\
\hline $\begin{array}{l}\text { Hospitalisation au cours des six mois précédents } \\
\text { Non } \\
\text { Oui }\end{array}$ & $\begin{array}{l}1 \\
3,9^{* *}\end{array}$ & $1,6-9,7$ \\
\hline $\begin{array}{l}\text { Co-morbidité chronique à l'inclusion } \\
\text { Non } \\
\text { Oui }\end{array}$ & $\begin{array}{l}1 \\
1,8^{* * *}\end{array}$ & $0,9-3,6$ \\
\hline
\end{tabular}

caractérisée par le fait d'avoir atteint le stade sida ou un taux de CD4 $<200 / \mathrm{mm}^{3}$ au cours de l'année précédente, est associée à une augmentation significative du risque de perte d'emploi chez les femmes (hazard ratio brut: 4,6 ; intervalle de confiance à $95 \%$ : $[1,8 ; 12,2])$, alors que, chez les hommes, la gravité de la maladie n'est pas associée au risque de perte d'emploi $(0,9$ $[0,5 ; 1,6])$. En tenant compte de cette hétérogénéité, il apparaît que, à caractéristiques comparables en termes de position sur le marché de l'emploi (âge, origine géographique, niveau d'éducation, catégorie socioprofessionnelle et statut d'emploi au moment du diagnostic du $\mathrm{VIH}$ ) et d'expérience de discriminations liées au VIH dans l'emploi, ce sont les femmes qui ont une maladie à VIH sévère qui ont un risque de perte d'emploi supérieur aux hommes (hazard ratio ajusté : 4,3 ; intervalle de confiance à $95 \%:[2,1 ; 8,7])$, alors que pour celles dont la maladie est moins avancée, il n'y a pas de différence hommes/femmes (1,0 $[0,6 ; 1,5]$ ) (figure 3 ).
Les analyses de la cohorte Primo et de l'enquête Vespa suggèrent donc de façon concordante que, à l'ère des traitements antirétroviraux, parmi les personnes séropositives qui travaillent, les femmes sont plus vulnérables au risque de perte d'emploi au cours de la maladie que les hommes. Cet excès de risque de perte d'emploi parmi les femmes semble être lié à une susceptibilité accrue de leur situation professionnelle aux épisodes de dégradation de l'état de santé. Différentes hypothèses peuvent être formulées pour expliquer ces résultats. Les femmes, souvent plus impliquées dans les responsabilités domestiques et familiales que les hommes, ont probablement davantage de difficultés à assumer des obligations professionnelles en plus des charges de leur foyer et de la prise en charge de leur maladie, en particulier quand elles doivent faire face à un problème de santé sévère. Les femmes vivent aussi plus souvent que les hommes en famille monoparentale (19,5\% versus $2,3 \%$ dans l'échantillon de l'enquête Vespa), une situation qui renforce encore ces 
Figure 3

Hazard ratio (HR) ajusté* de perte d'emploi au cours du temps, associé au fait d'avoir atteint un critère de gravité de la maladie à VIH dans l'année précédente, selon le sexe, parmi les 478 participants de l'enquête Vespa diagnostiqués à partir de 1996 et qui avaient un emploi au moment du diagnostic du VIH

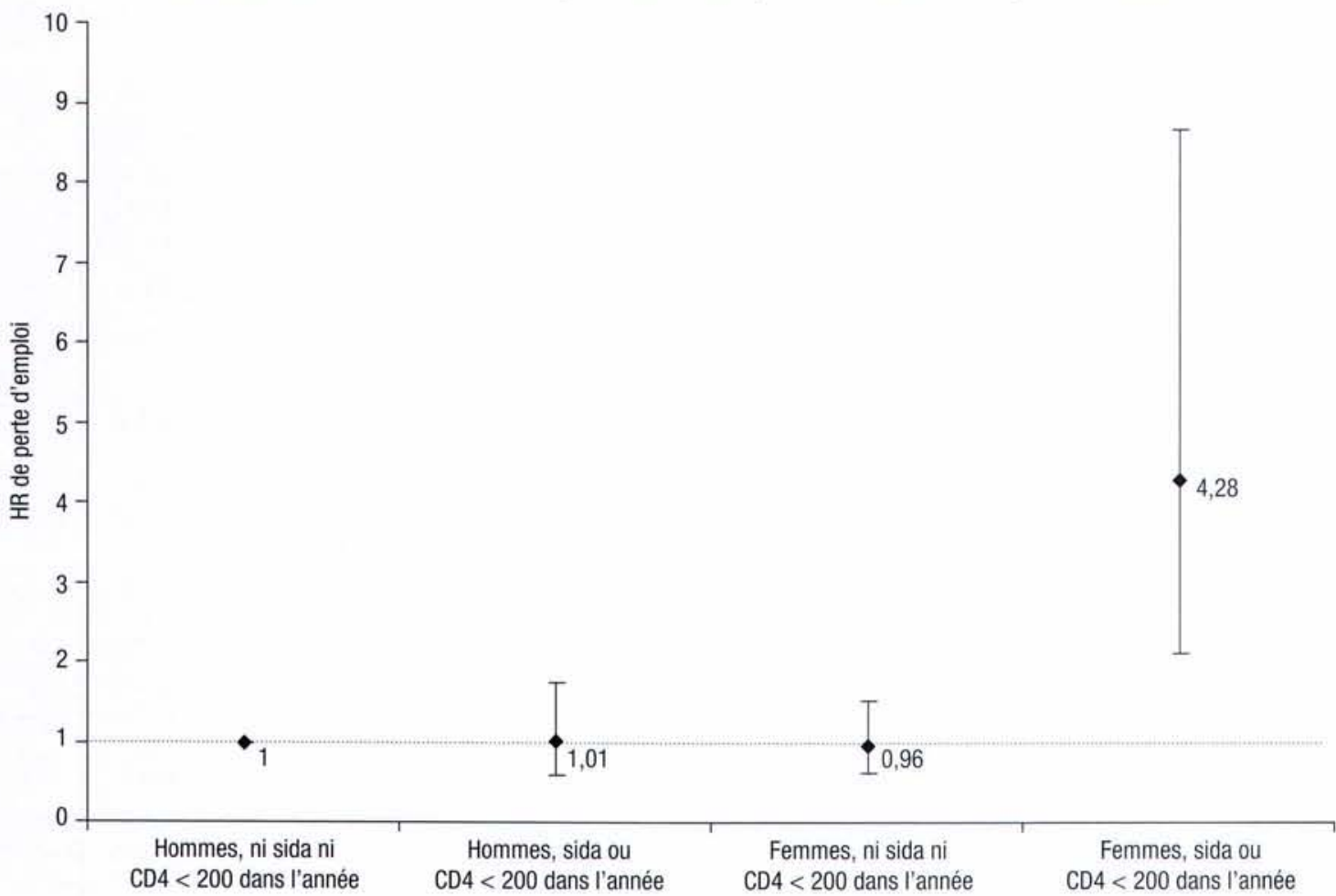

* Modèle de Cox multivarié, ajusté sur les caractéristiques sociodémographiques (âge, origine géographique, niveau d'éducation, catégorie socioprofessionnelle et statut d'emploi au moment du diagnostic) et sur l'expérience de discriminations liées au VIH dans l'emploi rapportée en 2003.

difficultés. Par ailleurs, les données que nous avons analysées ne permettent pas de déterminer dans quelle mesure le passage d'une situation d'emploi à une situation de non-emploi résulte d'un choix raisonné plutôt que d'un processus subi. II est possible que les différences que nous rapportons reflètent le fait que les femmes pourraient choisir plus souvent que les hommes d'arrêter de travailler quand elles sont malades, en particulier quand elles vivent en couple et qu'elles peuvent compter sur le revenu de leur conjoint. Cependant, des analyses complémentaires des données de l'enquête Vespa suggèrent que le sur-risque féminin de perte d'emploi, associé au fait d'avoir une maladie à VIH sévère existe non seulement pour les femmes qui vivent seules, mais également pour celles qui vivent en couple, rendant cette hypothèse peu probable pour expliquer nos résultats.

\section{Accès ou retour à l'emploi au cours de la maladie à VIH}

Au sein de l'échantillon de l'enquête Vespa, parmi les 961 participants qui ne travaillaient pas au moment du diagnostic de séropositivité, un tiers $(34,2 \%)$ avait accédé à un emploi au moment de l'enquête en 2003 , soit après une durée médiane de 8,6 ans après le diagnostic. Cette proportion varie à la fois selon le sexe et l'origine géographique des personnes : seulement $27,1 \%$ des femmes françaises ont accédé à un emploi entre le diagnostic et 2003 , contre $38,3 \%$ des femmes originaires d'un autre pays. Parmi les hommes, ces proportions atteignent respectivement $34,4 \%$ parmi les Français et $38,8 \%$ parmi les migrants.

En analyse multivariée, il apparaît que, en tenant compte de l'ancienneté du diagnostic du VIH, des caractéristiques de la position sur le marché de l'emploi (âge, niveau d'éducation, catégorie socioprofessionnelle, composition du foyer, taux de chômage dans la région de résidence) et des caractéristiques de l'état de santé (antécédents d'hospitalisation liée au VIH, antécédents de prescription de Bactrim ${ }^{\circledR}$, stade sida, taux de CD4, prescription d'un traitement antirétroviral, co-infection par le VHC, état de santé général perçu), les femmes migrantes accèdent plus fréquemment à un emploi au cours de la maladie à VIH comparées aux hommes français (odds ratio ajusté : 2,0 ; intervalle de confiance à $95 \%$ : $[1,0 ; 3,8])$. En revanche, les femmes françaises, de la 
Figure 4

Odds ratio ajusté ${ }^{\star}$ de retour/accès à l'emploi entre le diagnostic et 2003 pour les femmes comparé aux hommes parmi les 961 participants de l'enquête Vespa qui ne travaillaient pas au moment du diagnostic du VIH

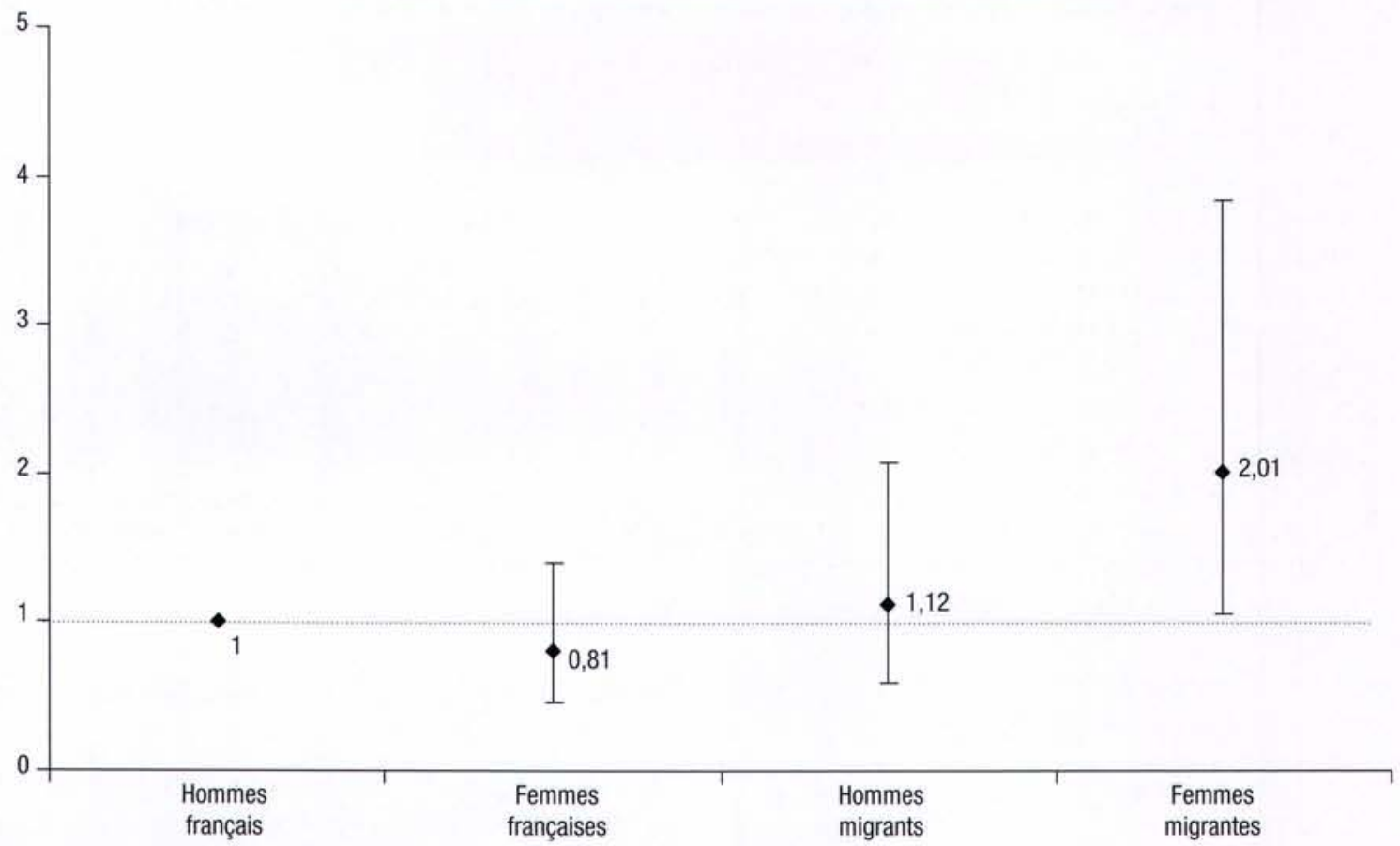

- Modèle de régression logistique ajusté sur les caractéristiques sociodémographiques (âge, niveau d'éducation, catégorie socioprofessionnelle, composition du foyer, taux de chômage dans la région de résidence), les caractéristiques de l'état de santé (antécédents d'hospitalisation liée au VIH, antécédents de prescription de BactrimX, stade sida, taux de CD4, prescription d'un traitement antirétroviral, coinfection par le VHC, état de santé générale perçu) et l'ancienneté du diagnostic du VIH.

même façon que les hommes migrants, accèdent aussi souvent à un emploi que les hommes français (figure 4).

Parmi les personnes immigrées, et tout particulièrement les femmes, le diagnostic de séropositivité survient souvent peu de temps après l'arrivée en France, à un moment où les possibilités d'accès à l'emploi sont limitées par des problèmes incluant le statut légal et la barrière de la langue : parmi les personnes immigrées de l'enquête Vespa, $48 \%$ des femmes étaient en France depuis moins d'un an au moment du diagnostic (alors que c'était le cas pour $22 \%$ des hommes) [20]. II en résulte un taux d'emploi particulièrement faible parmi les femmes migrantes au moment du diagnostic de séropositivité (35\% parmi les femmes immigrées, contre $60 \%$ parmi les hommes immigrés, $62 \%$ parmi les femmes françaises et $79 \%$ parmi les hommes français dans l'enquête Vespa). Le poids de telles barrières à l'accès à l'emploi liées à l'immigration récente diminue au fur et à mesure que la durée du séjour en France s'allonge, expliquant probablement la fréquence plus élevée d'accès à l'emploi parmi les femmes migrantes comparées aux autres groupes. Ainsi, des analyses complémentaires restreintes aux migrants de Vespa suggèrent qu'en tenant compte de l'ancienneté de l'arrivée en France au moment du diagnostic de VIH, la fréquence d'accès à l'emploi au cours de la maladie est comparable entre les hommes et les femmes. Les différences de taux d'accès à l'emploi que nous rapportons reflètent donc probablement le fait que les personnes qui ne travaillaient pas au moment du diagnostic de séropositivité avaient à cette période des caractéristiques très différentes sur le marché de l'emploi, selon qu'il s'agit de femmes migrantes ou non, plutôt qu'une réelle différence dans les chances d'accéder à un emploi liée au sexe et au statut migratoire.

Par ailleurs, les données de cette enquête mettent en évidence de nettes différences dans le type d'emploi auquel les personnes séropositives ont accès selon leur sexe et leur origine. Ainsi, parmi les femmes migrantes qui ont accédé à un emploi entre le diagnostic et 2003 , plus de la moitié $(52 \%)$ ont accédé à un emploi à statut précaire ; cette proportion atteint $39 \%$ parmi les femmes françaises, $26 \%$ parmi les hommes français et $27 \%$ parmi les hommes migrants.

\section{Conclusion}

Les résultats présentés dans ce chapitre suggèrent que, de la même façon qu'au sein de la population générale, parmi les personnes séropositives, les femmes ont un taux d'emploi significativement plus faible que les hommes. La proportion de femmes 
séropositives qui travaillent en 2003 atteint à peine une sur deux, même parmi celles dont le diagnostic de VIH a été porté au cours de la période d'accès aux traitements antirétroviraux hautement actifs (HAART), ou peu avant et ce, alors qu'il s'agit d'une population constituée principalement de personnes en âge de travailler. Au niveau global, l'écart de taux d'emploi avec la population générale est du même ordre pour les hommes et les femmes séropositifs, suggérant un impact comparable de la maladie à VIH sur l'emploi pour les deux sexes. Toutefois, quand on s'intéresse aux processus dynamiques d'entrées/sorties du travail, il apparaît certaines différences entre les hommes et les femmes. En effet, la situation professionnelle des femmes, plus précaire que celle des hommes ( $27 \%$ des femmes de l'enquête Vespa occupaient un emploi à statut précaire au moment du diagnostic de $\mathrm{VIH}$, contre $18 \%$ des hommes) et qui s'ajoute à des charges domestiques et familiales souvent plus lourdes, apparaît plus déstabilisée par les effets de la maladie, en particulier au moment où celle-ci devient sévère. De plus, si certaines femmes, en particulier les migrantes, qui ont des taux d'emploi particulièrement faibles au moment où la maladie est diagnostiquée, en lien avec leur arrivée récente en France, parviennent à accéder à un emploi après ce diagnostic (condition nécessaire pour sortir de la situation de grande détresse socio-économique dans laquelle elles se trouvent à leur arrivée), elles n'ont cependant souvent accès qu'à des emplois précaires. L'infection par le VIH elle-même, mais aussi les caractéristiques sociodémographiques et professionnelles pèsent donc de façon concomitante sur la situation d'emploi des personnes atteintes et rendent les femmes séropositives particulièrement exposées aux difficultés d'emploi.

\section{Références bibliographiques}

1. Greenwald HP, Dirks SJ, Borgatta EF, et al. Work disability among cancer patients. Soc Sci Med 1989; 29(11) : 1253-9.

2. Bartley M, Sacker A, Clarke P. Employment status, employment conditions, and limiting illness : Prospective evidence from the British household panel survey 1991-2001. J Epidemiol Community Health 2004 ; 58(6) : 501-6.

3. Moser KA, Fox AJ, Jones DR. Unemployment and mortality in the OPCS Longitudinal Study. Lancet 1984 ; $2(8415)$ : 1324-9.

4. Sorlie PD, Rogot E. Mortality by employment status in the National Longitudinal Mortality Study. Am J Epidemiol 1990; 132(5) : 983-92.
5. Voss M, Nylen L, Floderus B, Diderichsen F, Terry PD. Unemployment and early cause-specific mortality : a study based on the Swedish twin registry. Am J Public Health $2004 ; 94(12):$ 2155-61.

6. Association AIDES. Enquête auprès des personnes séropositives et de leurs proches utilisant les services de l'association AIDES : synthèse des résultats de l'enquête. 1999.

7. Cunningham WE, Andersen RM, Katz MH et al., The Impact of competing subsistence needs and barriers on access to medical care for persons with Human Immunodeficiency Virus receiving care in the United States. Med Care $1999 ; 37(12): 1270-81$.

8. Dray-Spira R, Lert F, Marimoutou C, Bouhnik AD, and Y. Obadia Y. Socioeconomic conditions, health status and employment among persons living with HIV/AIDS in France in 2001. AIDS Care 2003; 15(6) : 739-48.

9. Ezzy D, De Visser R, Bartos M. Poverty, disease progression and employment among people living with HIV/AIDS in Australia. AIDS Care 1999; 11(4) : 40514.

10. Ezzy D, De Visser R, Grubb I, McConachy D. Employment, accommodation, finances and combination therapy: the social consequences of living with HIV/AIDS in Australia. AIDS Care 1998. 10 Suppl 2: S189-S99.

11. Fabre G, Tchobanian R. Les tensions actuelles entre le VIH et l'emploi. Sc Soc Santé 2001 19(3) : 43-67.

12. Fleishman JA. Transitions in insurance and employment among people with HIV infection. Inquiry $1998 ; 35$ (Spring) : 36-48.

13. Goldman DP, Bao Y. Effective HIV treatment and the employment of HIV(+) adults. Health Serv Res 2004. 39(6) : 1691-712.

14. Kupek E, Dooley M, Whitaker L, Petrou S, Renton A. Demographic and socioeconomic determinants of community and hospital services costs for people with HIV/AIDS in London. Soc Sci Med 1999; 48(10) : 1433-40.

15. Rabkin JG, McElhiney M, Ferrando SJ, Van Gorp W, Lin SH. Predictors of employment of men with HIV/AIDS : a longitudinal study. Psychosom Med $2004 ; 66(1): 72-8$.

16. Vitry-Henry L, C. Penalba I, Beguinot, Deschamps F. Relationships between work and HIV/AIDS status. Occup Med $1999 ; 49(2)$ : 115-6.

17. Insee. Femmes et hommes : regards sur la parité. Paris : Insee, 2004, 176 p.

18. Desquilbet L, Deveau C, Goujard C et al. Increase in at-risk sexual behaviour among HIV-1-infected patients followed in the French PRIMO cohort. AIDS $2002 ; 16(17): 2329-33$.

19. Peretti-Watel P, Riandey B, Dray-Spira R et al. Comment enquêter la population séropositive en France? : I'enquête Anrs-Vespa 2003. Population 2005; $60(4): 525-50$

20. Lert F, Obadia Y et l'équipe de l'enquête Vespa. Comment vit-on en France avec le VIH/sida ? Pop et Sociétés 2004(406). 